\title{
Integrated Mutation Strategy with Modified Binary PSO Algorithm for Optimal PMUs Placement
}

\begin{abstract}
Optimal phasor measurement units (PMUs) placement refers to the strategic placement of PMUs to achieve the full observability of power systems with a minimum number of PMUs. A strategic placement is needed because of the economic or technical restriction that hinders the deployment of PMUs on every bus. A modified version of the binary particle swarm optimization (BPSO) method is proposed in this paper by integrating a mutation strategy and the $\mathrm{V}$-shaped sigmoid function for placing the PMUs that maintains the full power system observability in the presence of zero-injection bus, single PMU loss and PMU's channel limits while maximizing the measurement redundancy. The solution that has the highest measurement redundancy was selected as the best placement of PMUs. The use of mutation strategy and $\mathrm{V}$-shaped sigmoid function in this paper improves the population diversity, thereby minimizing the chance of the particles being trapped in the local optima, consequently leading to a quality solution. In order to validate its effectiveness, the results obtained by the proposed method are compared with other published techniques to demonstrate the accuracy and validity of the proposed technique. The results of the IEEE 300bus system show that the proposed method effectively managed to reduce the number of PMUs needed.
\end{abstract}

Index Terms - binary particle swarm optimization, PMU, mutation, smart grid

\section{INTRODUCTION}

$\mathrm{P}$ hasor measurement unit (PMU) is a measurement device equipped with the Global Positioning System (GPS), hence, the measurement data provided are in real-time. As reported in the post-mortem report of the North American blackout in 2003, the lack of real-time measurement provided through the state estimation at that time prevented the operating engineers to correctly execute the contingency plans they had in place [1]. Since the real-time measurements of the voltage and branch currents of the bus can be obtained when a PMU is installed on that bus, the state of the power system can be identified based on the direct measurements provided by the PMUs if they are all installed at every bus [2]. However, the PMUs are not necessarily needed to be installed at every bus since the voltage and currents of the bus can be obtained through indirect measurements using the Ohm's Law and Kirchhoff's Current Law (KCL) [2]. In general, the minimum number of PMUs required is in the range of $20-30 \%$ of the number of system buses [3]. Hence, the objective of the optimal PMUs placement (OPP) problem is mainly focused on finding the minimum number of PMUs required in a power system and its location in order to make the power system observable [4].
Recently, numerous optimization methods have been proposed to solve the OPP problem. The integer linear programming (ILP) method is widely used for solving the OPP problem [5]-[8] since it is capable of solving the OPP problem in a very short time. The exhaustive search (ES) method [9][11] and heuristic algorithms such as simulated annealing (SA) [12], genetic algorithm (GA) [13], [14], firefly algorithm (FA) [15], tabu search [16], differential evolution (DE) [17], [18], and particle swarm optimization (PSO) through a binary variant called binary PSO (BPSO) [19]-[25] have shown that they are also capable of finding the optimal placement of PMUs. In these existing studies, many constrained factors such as the effect of the zero-injection bus (ZIB), conventional measurement, a single PMU loss, line outage and PMU's channel limits are considered while solving the OPP problem. Among these factors, the PMU's channel limits is rarely considered while using the heuristic algorithms. The GA [13], [14] and FA [15] are the only heuristic algorithms considering the PMU's channel limit when solving the OPP problem.

The BPSO algorithm is easy to implement and can converge to the optimal solution [26]. However, it also tends to be stuck in the local optima especially when dealing with complex multimodal functions. In order to prevent the algorithm from being stuck in local optima in solving the OPP problem, various methods have been proposed to improve the performance of the algorithm. Many techniques such as modifying the velocity update equation [20]-[22], the exclusion of some buses from the placement of PMUs [23], and also incorporating techniques from other heuristic algorithms into BPSO algorithm [25] were among those being proposed to solve the OPP problem using the BPSO algorithm. Finally, the channel limit constraint was never considered while using the BPSO algorithm.

The importance of the measurement redundancy as one of the most crucial parameters for the secure monitoring of the power systems has been highlighted in the recent years [27]. Therefore, it is used to differentiate the quality of each placement set of PMUs, where the best optimal result was chosen based on the placement set of PMUs that has the highest measurement redundancy. Recent papers showed that there is still room for further investigation in terms of measurement redundancy for the BPSO algorithm since the results obtained from the existing methods using the BPSO algorithm are not the optimal solution [27], [28].

In this paper, a novel BPSO algorithm with a new integrated mutation strategy to solve the OPP problem while considering the PMU's channel limit, ZIB and single PMU loss is presented. 
In contrast to the existing methods, this paper presents the use of V-shaped sigmoid function and a new position update rules while solving the OPP problem. The mutation strategy is also proposed to compliment the $\mathrm{V}$-shaped sigmoid and the position update rules. The mutation strategy was designed to refine the local search of the algorithm more effectively and at the same time help to instigate the particles to move from their current location to prevent them from being stuck in the local optima. In addition, for the single PMU loss, this paper proposed a fitness function that integrates the problem constraint into the fitness function, for ease of implementation and at the same time reduce the computation burden. Aside from minimizing the number of PMUs used to achieve a complete observability of the power system, the measurement redundancy was also considered such that the placement set of PMUs will be of the highest quality. In order to determine the measurement redundancy, the bus observability index (BOI) and system observability redundancy index (SORI) were used. BOI refers to the number of times a bus was observed by placement of PMUs, while SORI refers to the sum of BOIs [6]. The placement set of PMUs that has a higher SORI value implies that it is more reliable and robust against contingencies. Hence, the placement set of PMUs that has the highest value of SORI indicates that it is the best optimal result.

This paper is organized into five sections. Section II explains the PMU placement rules for problems under consideration and how each case considered in this paper was formulated. Section III describes the BPSO algorithm and the fitness function used in this paper. Section IV explains the proposed method used to solve the optimal PMU placement. The simulation results and discussion are covered in Section $\mathrm{V}$, where the results are also compared with prior studies to evaluate its effectiveness. Section VI concludes the paper.

\section{PMU PLACEMENT FoRMULATION}

Observability analysis used to analyze the power system can be categorized into two categories: numerical and topological observabilities. In the numerical observability, the full observability of a system is verified based on whether the Jacobian matrix was of full rank. However, it involves huge matrix manipulation and is computationally expensive [16]. Meanwhile, the topological observability analysis is based on whether a spanning tree of full rank can be constructed where each branch can be observed by direct measurements or calculations [22]. Therefore, this paper used the topological observability to identify a power system as observable.

For a power system to be identified as fully observable, the voltage for all its buses must be known. In order to decide whether a bus is observable, its voltage must be known either through direct measurements or calculation by using other known parameters. A PMU installed bus can have its voltage and branch current measured directly by PMU. Meanwhile, by using indirect measurements, buses that are neighbors to the PMU installed bus can have their voltage values known through calculations using the Ohm's law. Hence, with direct and indirect measurements, a power system can be identified as observable based on the following rules [5]:
1. If the voltage at one end and its branch current are known, the voltage at the other end can be calculated.

2. In a situation where the voltage of both ends is known, the branch current for the branch that connects both ends can be calculated.

The following discussions expand the PMU formulation to integrate the specific characteristics of the power system in this paper. Three characteristics - zero-injection bus, single PMU loss, and PMU's channel limits - are considered in this paper for solving the OPP problem.

\section{A. Effect of zero-injection bus}

A ZIB is a bus that has no power or load injected into it. Thus, the sum of flows on all branch currents associated with ZIB is zero according to the Kirchhoff's current law (KCL). The following rules are considered in addition to the rules mentioned earlier when solving the OPP problem for power systems considering ZIB [5]:

1. If all buses adjacent to an observable ZIB are observable except one, by applying KCL at the ZIB, it will make the unobservable bus as observable.

2. An unobservable ZIB can be determined as observable, if every bus adjacent to it is observable, by applying KCL at the ZIB.

3. A group of unobservable ZIBs can be determined as observable if every bus connected to them is observable.

From the above rules, it can be concluded that when considering ZIB in a power system, the ZIB and buses that are adjacent to ZIB must be observable except one to ensure that it is observable through indirect measurement. Therefore, the number of PMUs needed is reduced since there are fewer buses that need to be observed.

\section{B. Effect of single PMU loss}

The power system may be exposed to several contingencies in the event when power system uncertainty happens. Hence, it is crucial that the observability of the power system is not affected in any contingency. With regard to PMU, according to the N-1 criterion, the optimal PMU placement problem should be able to endure the single PMU loss, in which the observability of the power system must be maintained [29]. Therefore, every bus must be observed by at least two PMUs to ensure a bus remains observable should one of the PMUs malfunction.

\section{Effect of channel limits}

Most studies assumed that PMU has unlimited channels. However, in practical situations, there is a fixed channel [27]. In the case where fixed channel is considered, the following approach can be used. For cases considering channel limits, consider bus 2 in the 7-bus system.

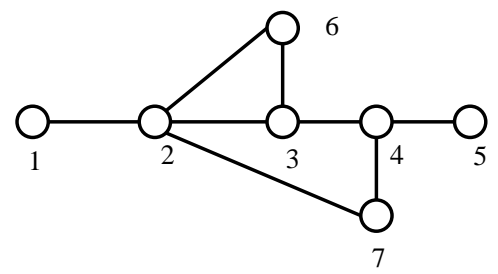

Fig. 1 7-bus system 
In order to observe bus 2 and its branch current with a single PMU, a PMU with the minimum of five channels is needed. One channel is used to measure the voltage on the bus, and four more channels are used to measure the branch currents $(2-1,2-$ $3,2-6,2-7)$. If the PMU has fewer channels, the possible combinations for each bus need to be identified. The situation can be simplified as follows [30]:

$$
B R_{i}=\left\{\begin{array}{cc}
B C_{i} & \text { if } L \leq B I_{i} \\
1 & \text { if } B I_{i}<L
\end{array}\right.
$$

where $B R_{\mathrm{i}}$ is the number of branch combinations for bus $i, L$ is the channel limit, $B I_{i}$ is the number of buses adjacent to bus $i$, $B C_{i}$ is the number of possible combinations of $L$ out of $B I_{\mathrm{i}}$ where it can be defined as:

$$
B C_{i}=\frac{B I_{i} !}{\left(B I_{i}-(L-1)\right) !(L-1) !}
$$

Now that the value of $B R_{\mathrm{i}}$ is known for all buses, a binary connectivity matrix based on the possible combinations for each bus is defined as matrix $[H]$, where bus $i$ will have $B R_{\mathrm{i}}$ rows. Assuming a channel limit of 3 is set for the PMUs, the total of possible combinations for the 7-bus system is 16 . Accordingly, matrix $[H]$ in this case can be defined as in (4).

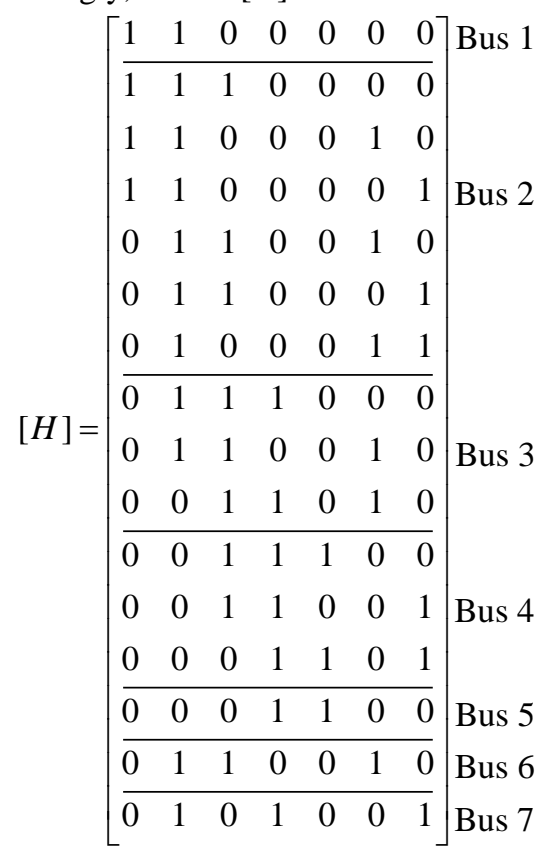

The number of branch combinations above can be reduced by considering the duplicate branch and the radial bus. For example, row 5 (R5) and row 9 (R9) carry the same branch combination. Thus, removing one of the combinations does not impact the topological observability. However, it also means to monitor the corresponding branch, the option is only restricted to one. In the case of considering the radial bus, row 14 (R14) can be eliminated since placing a PMU at bus 5 restricts the observability to only two buses, hence a waste of the extra channel that the PMU has.

\section{BINARY PARTICLE SWARM OPTIMIZATION}

PSO is a population-based optimization method inspired by the social behavior of bird flocking or fish schooling. It begins with the random initialization of a population (swarm) of individuals (particles) in the search space [31]. These particles will fly through the search space based on their experience and other particles' experiences. The particle that holds the best experience will be promoted as the global best where the other particles will be attracted to it. The particles will continue exchanging their experience, and a new global best may be nominated throughout the algorithm. The optimization process ends based on the defined stopping criteria. In this paper, the number of maximum iterations is set as the stopping criteria.

In the conventional PSO, the current velocity holds by each particle and their previous position are used to decide the next position for each particle. The velocity indicates the next trajectory of the particle from its previous position. The value of velocity, $v$, and position vector, $x$, is updated accordingly by using the following equations:

$$
\begin{gathered}
x_{i j}^{t+1}=x_{i j}^{t}+v_{i j}^{t+1} \\
v_{i j}^{t+1}=K\left[v_{i j}^{t}+c_{1} r_{1}\left(\text { pbest }_{i j}^{t}-x_{i j}^{t}\right)+c_{2} r_{2}\left(\text { gbest }^{t}-x_{i j}^{t}\right)\right]
\end{gathered}
$$

where $K$ is the constriction factor that can be defined as follows [32]:

$$
K=\frac{2}{\left|2-\phi-\sqrt{\phi^{2}-4 \phi}\right|}, \text { where } \phi=c_{1}+c_{2}, \phi>4
$$

$x_{\mathrm{ij}}(t)$ and $x_{\mathrm{ij}}(t+1)$ are the position vectors of the $i^{\text {th }}$ particle in the $j^{\text {th }}$ dimension at iteration $t$ and $t+1$, respectively, while $v_{\mathrm{ij}}(t+1)$ indicates the velocity vector of the particle at iteration $t+1 ; c_{1}$ and $c_{2}$ are the two acceleration constants, where it is suggested that the values for both constants are equal to 2.05 [32]; $r_{1}$ and $r_{2}$ are two random numbers that are uniformly distributed within the range of $[0,1]$. Meanwhile, pbest $t_{i j}(\mathrm{t})$ indicates the best position of particle $i^{\text {th }}$ in the $j^{\text {th }}$ dimension it has found so far while gbest $(\mathrm{t})$ indicates the best position in the swarm at iteration $t$. The values for pbest and gbest are determined according to the best fitness value it holds.

In regard to the PMU placement problem, a discrete PSO variant named binary PSO (BPSO) is used. It was developed by Kennedy and Eberhart to operate on the discrete binary variables [33]. In the conventional PSO, the velocity vector is used as the degree of change for particles' position vector $x$. However, in BPSO, it is used as the probability threshold to change the position vector $x$ to be in a state of 0 or 1 . Based on the value of the velocity vector, the real values of velocities are transformed to the probability values in the interval of $[0,1]$ by using the sigmoid function.

$$
\begin{gathered}
\operatorname{sig}\left(v_{i j}\right)=\frac{1}{1+e^{v_{i j}}} \\
x_{i j}^{t+1}= \begin{cases}1 & \text { if } \text { rand } \leq \operatorname{sig}\left(v_{i j}\right) \\
0 & \text { otherwise }\end{cases}
\end{gathered}
$$

Constraint (9) is then used to update the position vector $x$ replacing (5) in the conventional PSO. The parameter rand denotes a random number between $[0,1]$. It can be said from (9) that the probability of getting one is $\operatorname{sig}\left(v_{i j}\right)$ while the probability of getting zero is 1-sig $\left(v_{i j}\right)$. Hence, it is important to note how significantly the value of the velocity vector influences the position vector $x$ in BPSO. 
In this paper, the main objective is to find the minimum number of PMUs and to maximize the measurement redundancy while ensuring the full observability of a power system. Therefore, the fitness function, $Z$ which was introduced in [19] is used in this paper to evaluate every particle in the swarm. The minimization of fitness function is formulated as follows:

$$
Z=\min \left\{\left(w_{1} \times N_{o b s}\right)+\left(w_{2} \times N_{P M U}\right)+\left(J_{1} \times C\right)\right\}
$$

where parameters $w_{1}, w_{2}$, and $C$ are three weight values. $N_{\text {obs }}$ signifies the number of observable buses, $N_{P M U}$ is the total number of PMUs and $J_{1}$ is the measurement redundancy that can be defined as follows:

$$
J_{1}=(M-A X)^{T} \times(M-A X)
$$

where $A$ is a binary connectivity matrix, whose entries can be defined as follows:

$$
A_{i, j}= \begin{cases}1 & \text { if } i=j \\ 1 & \text { if bus } i \text { and } j \text { are connected } \\ 0 & \text { otherwise }\end{cases}
$$

The binary decision variable vector, $X$, is defined as:

$$
x_{i}= \begin{cases}1 & \text { if a PMU is installed at bus } i \\ 0 & \text { otherwise }\end{cases}
$$

$M$ is the desired value of measurement redundancy which entries are defined as follows:

$$
M=\left[\begin{array}{lllll}
m_{1} & m_{2} & m_{3} & \cdots & m_{N}
\end{array}\right]_{1 \times N}^{T}
$$

If the desired value for measurement redundancy is 2 , the element of $M$ will be set to 3 . Hence, the difference between the actual and desired number of times the bus is observed is computed using vector $(M-A X)$. In all, it means that minimizing this difference is equivalent to maximizing the measurement redundancy. Thus, the value of parameters $w_{1}, w_{2}$ and $C$ must be comparable in magnitudes. Note that, the maximum value for each element in $M$ depends on the number of bus incident to it. For example, bus 1 in Fig. 1 is only connected to bus 2 , therefore, the maximum value that can be set for bus 1 is two (where PMU can only be placed either at bus 1 or bus 2 or both).

\section{Proposed Method Formulation}

This paper proposed a mutation strategy, which is integrated with the V-shaped sigmoid and the new position update rules to solve the OPP problem.

\section{A. V-shaped sigmoid function}

The BPSO algorithm offers quick convergence speed but the particles are tend to trap themselves in the local optima [34]. The sigmoid function (8) and the position update (9) introduced for the BPSO algorithm were claimed to be the reason for it being trapped in the local optima because it encourages the particles to change position at every opportunity they get [35]. Fig. 2 illustrates the graph for $\mathrm{S}$-shaped sigmoid and V-shaped sigmoid function where $-5 \leq v \leq 5$. The $\mathrm{S}$-shaped sigmoid refers to the sigmoid function (8). For the S-shaped sigmoid, in the case where $v=0$, the probability of changing bit is $\operatorname{sig}(v)=0.5$ as can be seen in Fig. 2.
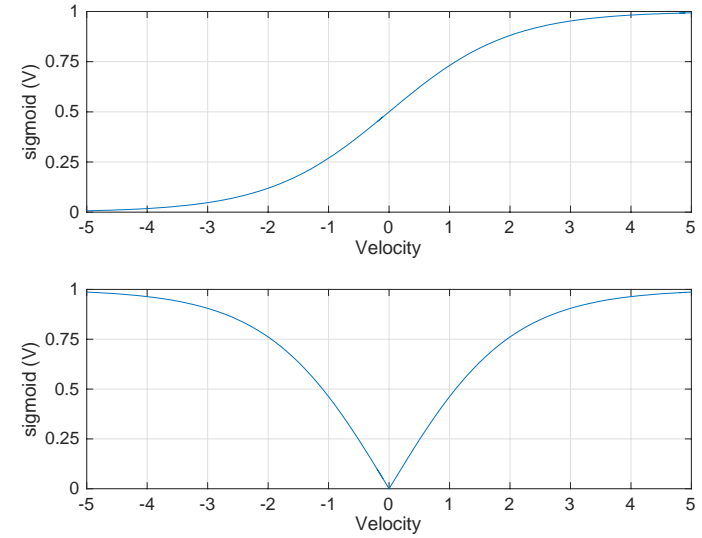

Fig. 2 S-shaped sigmoid (top) and V-shaped sigmoid (bottom)

Based on the position updating rules (9), it will force the particles to take the value of 0 or 1 . This means that the particle can quickly be at state 1 in the current iteration and 0 in the next iteration and vice versa. Moreover, this prevented the particles from converging, which led to the random positioning of particles - hence, this hindered the particles from exploiting their current position that most of the time led to an infeasible solution. The V-shaped sigmoid function was proposed to overcome this issue, where it can be defined as follows [36]:

$$
\operatorname{sig}\left(v_{i j}\right)=2 \times\left|\frac{1}{1+e^{-v_{i j}(t+1)}}-0.5\right|
$$

Then, the probability values obtained from (15) are used to update position vectors $x$ as follows:

$$
x_{i j}^{t+1}= \begin{cases}\left(x_{i j}^{t}\right)^{1} & \text { if } \operatorname{rand}<\operatorname{sig}\left(v_{i j}\right) \\ x_{i j}^{t} & \text { otherwise }\end{cases}
$$

In contrast to the behavior of the S-shaped sigmoid and position update rules proposed in the conventional BPSO, the position update rules (16) and the $\mathrm{V}$-shaped sigmoid shown in Fig. 2 stimulate particles to flip the current positions they are in to their complements when the velocity values are high and remain in their current positions when their velocity values are low [36].

\section{B. Mutation}

Population diversity shows the difference among individuals. Therefore, by maintaining population diversity, the possibility of finding a final reasonable solution is great [37]. However, it is important that the balance between exploration and exploitation is maintained to ensure the search space is well scrutinized for the best solution. For the optimization algorithms that rely on the population to find a solution such as PSO, the mutation is adopted in the algorithm itself where changes are made to the particles so that the next iteration of particles explores the area in the landscape that was proven good for the prior iteration [10]. In PSO, mutation is achieved through random number $r_{1}$ and $r_{2}$, where the mutation magnitude is determined by the past velocity, pbest and gbest as derived in (6). Consequently, in the situation where prior experience is not reliable or bound to be trapped in local optima, the particles will not be able to mutate themselves to increase diversity. Hence, an external influence that can modify the position or velocity of the particle outside the velocity and the update rules may help in improving the chance of producing 
quality solutions [25], [38], [39]. Therefore, in this paper, a mutation strategy is proposed to help refine the local search of the algorithm and subsequently help to prevent the algorithm from being stuck in the local optima. The proposed mutation strategy will make changes to the position of the particle by flipping the bit of 0 to 1 , or 1 to 0 as illustrated in Fig. 3 as below.

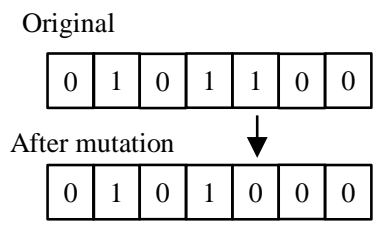

Fig. 3 Basic mutation

The following is the list of procedures for the mutation strategy proposed in this paper for solving the OPP problem:

Step 1: Select the particles to mutate based on the average number of PMUs from the pbest of the particles and store them in an array called the particleMutation.

Step 2: Find the fitness values for all elements in particleMutation. Discard all but one particle that shared the same fitness value.

Step 3: For each element in the particleMutation, find the bus/buses that have the maximum BOI and store it in an array called the busList and find all buses adjacent to the busList and store it in an array called the busToMutate.

Step 4: Loop through the busToMutate, and at every iteration, mutate each bus in the list by complementing its current state. For example, if it is 0 , flip it to 1 . In addition, place a PMU at a bus that adjacent to a radial bus and remove any PMU placed at a radial bus. Store each mutated solution in an array called the mutatedList.

Step 5: Evaluate each solution in the mutatedList by using fitness function and store the value in an array called the tempFitness.

Step 6: Once finished, find the optimal solution based on the TempFitness. Compare the fitness function of the optimal solution with pbest fitness for the particle under scrutiny. If it is better, replace it. If it is not, discard it. Repeat steps 3-6 for all elements in the particleMutation

The main principal behind the mutation strategy is to evaluate the possible solutions around the pbest of selected particles. If a better solution is found around the current pbest, it will be replaced by the better solution. Otherwise, the pbest remains for the next iteration.

The mutation strategy is executed after pbest for each particle is updated as shown in Fig. 4. In order to select the candidate particles, the average number of PMUs from all the pbest in the current iteration is used. Any pbest which carries the same number of PMUs to the average number of PMUs are gathered.

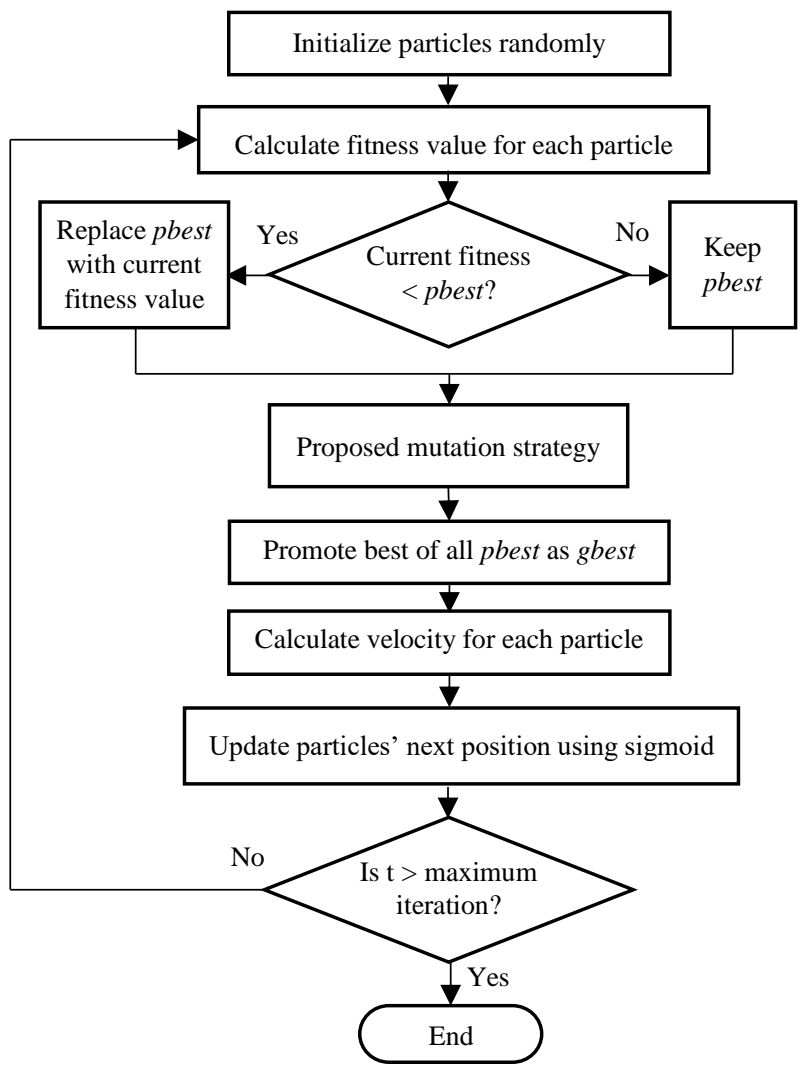

Fig. 4 Flowchart of BPSO algorithm

In order to find the possible solutions around the pbest, the buses that are neighbors around the bus that has the highest BOI are mutated, including the bus itself. The bus that has the highest BOI tends to have a cluster of PMUs placed around it. Therefore, applying mutation to these buses may help in finding the better PMU around the bus.

The mutation strategy also considered the presence of radial bus in a power system by having PMUs pre-assigned to the bus adjacent to the radial bus and prohibited PMU from being placed by the algorithm to the radial bus. Since there is only one bus connected to the radial bus, placing a PMU at a radial bus limits the PMU's coverage to only two buses (itself and its neighbor) whereas placing a PMU at the bus that is located next to a radial bus will ensure more bus can be made observable and increase the network coverage of the power system. However, if the neighbor to the radial bus is a ZIB, a PMU does not need to be pre-assigned because the radial bus could be made observable through pseudo-measurement. This approach encourages particles to find more feasible solutions during the searching process and at the same time improves the algorithm convergence rate.

The consequence of the mutation of each bus as described earlier will be evaluated and compared to find the optimal result from the mutation process, where, if the result of the mutation process is better, it will replace the current pbest. Otherwise, the current pbest will remain. The integration of the mutation strategy also presents a number of extra fitness evaluations, which consequently increases the computational cost and time. Therefore, to overcome this issue, if there are more than one pbest that have the same fitness value, only one pbest will be considered for the mutation process. This is because the pbest 
of the same fitness value carry the same PMUs placement, hence, through the mutation strategy, the results of the mutation will be the same. Hence, discarding all but one pbest for the mutation process help to reduce the computation cost and time.

The use of the V-shaped sigmoid and position update rules also means that if the particle no longer carry any velocity, it will never be able to change its position since it will remain in their current position as shown in (16). Therefore, the proposed mutation strategy will be able to instigate the particle to jump out from its current position if there is a better solution than it currently holds.

\section{Single PMU loss}

In order to integrate the single PMU loss constraint, this paper expands the fitness function proposed in [19] by formulating the problem in fitness function as follows:

$$
Z=\min \left\{\left(w_{1} \times N_{o b s}\right)+\left(w_{2} \times N_{P M U}\right)+\left(w_{3} \times N_{L}\right)+\left(w_{4} \times S\right)\right\}
$$

where,

$$
\begin{gathered}
N_{L}=|\{x \in D \mid x<0\}| \\
D=A X-b, \text { where } b=\left[\begin{array}{lllll}
2 & 2 & 2 & \cdots & 2
\end{array}\right]_{1 \times N}^{T} \\
S=\sum_{i=1}^{N} D_{i}
\end{gathered}
$$

In (17), $w_{3}$ and $w_{4}$ are two weight values. The parameter $N_{L}$ refers to the number of buses that are not being observed twice by the PMUs placement and $S$ is the measurement redundancy used when considering the single PMU loss. Therefore, the parameters $J_{1}$ and $C$ used for measurement redundancy in (10) are replaced with $S$ when considering the single PMU loss. In (19), vector $b$ refers to the binary vector variables that are equivalent to the number of times a bus needs to be observed. For the single PMU loss, the value is set as 2 since each bus needs to be observed at least twice.

\section{SimULATION RESUlTS AND DISCUSSION}

The proposed method was tested on IEEE 14-bus, 24-bus, 30-bus, 39-bus, 57-bus, 118-bus and 300-bus systems and simulated for five cases - (i) base case, (ii) ZIB, (iii) single PMU loss, (iv) channel limit for base case, (v) channel limit considering ZIB.

The value of each parameter used in the proposed method is given in Table I. MATLAB software was used to simulate the proposed method, and the technical specification of the computer used was Intel core i5 $2.5 \mathrm{GHz}$ with $8 \mathrm{~GB}$ of RAM.

TABLE I: The value of each parameter used in simulation

\begin{tabular}{lc}
\hline \multicolumn{1}{c}{ Parameters } & \begin{tabular}{c} 
Value \\
\hline Number of particles
\end{tabular} \\
$\begin{array}{l}4 * \text { Number } \\
\text { of bus }\end{array}$ \\
\hline Number of iterations & 2000 \\
\hline Weight value for the number of bus observed, $\boldsymbol{w}_{\mathbf{1}}[\mathbf{1 9}]$ & -2 \\
\hline Weight value for the number of PMUs, $\boldsymbol{w}_{\mathbf{2}}[\mathbf{1 9}]$ & 1 \\
\hline $\begin{array}{l}\text { Weight value for bus that is not being observed twice by } \\
\text { PMUs placement, } \boldsymbol{w}_{\mathbf{3}}\end{array}$ & 2 \\
\hline $\begin{array}{l}\text { Weight value for single PMU loss measurement } \\
\text { redundancy, } \boldsymbol{w}_{\mathbf{4}}\end{array}$ & -0.02 \\
\hline Weight value for the measurement redundancy, $\boldsymbol{C}$ [19] & 0.01 \\
\hline
\end{tabular}

Table II shows the number of PMUs, $N_{\mathrm{PMU}}$, the PMUs placement and the SORI value for every bus systems tested for the base case. For the case considering the ZIB, the PMUs placement and its SORI value is given in Table III. As can be seen from Tables II and III, the number of PMUs required to achieve complete observability of a power system are reduced when considering the ZIB compared to the base case. As noted earlier, it is because one of the neighbors to the ZIB can be indirectly measured when applying KCL at the ZIB, hence,

\begin{tabular}{|c|c|c|c|}
\hline $\begin{array}{l}\text { IEEE Bus } \\
\text { System }\end{array}$ & $N_{\text {PMU }}$ & Locations of PMUs & SORI \\
\hline 14-Bus & 4 & $2,6,7,9$ & 19 \\
\hline 24-Bus & 7 & $2,3,8,10,16,21,23$ & 31 \\
\hline 30-Bus & 10 & $2,4,6,9,10,12,15,19,25,27$ & 52 \\
\hline 39-Bus & 13 & $\begin{array}{l}2,6,9,10,13,14,17,19,20,22,23,25, \\
29\end{array}$ & 52 \\
\hline 57-Bus & 17 & $\begin{array}{l}1,4,6,9,15,20,24,28,30,32,36,38 \\
41,47,51,53,57\end{array}$ & 72 \\
\hline 118-Bus & 32 & $\begin{array}{l}3,5,9,12,15,17,21,25,28,34,37,40, \\
45,49,52,56,62,64,68,70,71,76,79, \\
85,86,89,92,96,100,105,110,114\end{array}$ & 164 \\
\hline 300-Bus & 87 & $\begin{array}{l}1,2,3,11,12,15,17,20,23,24,26,33, \\
35,39,43,44,49,55,57,61,62,63,70, \\
71,72,74,77,78,81,86,97,102,104, \\
105,108,109,114,119,120,122,124, \\
130,132,133,134,137,139,140,143, \\
153,154,159,164,166,173,178,184, \\
188,194,198,204,208,210,211,214, \\
217,223,225,229,231,232,234,237, \\
238,240,245,246,249,9002,9003, \\
9004,9005,9007,9012,9021,9023, \\
9053\end{array}$ & 432 \\
\hline
\end{tabular}
reduced the number of bus that needs to be observed directly by the PMUs.

TABLE II Result for base case

TABLE III Result for case considering ZIB

\begin{tabular}{cclc}
\hline $\begin{array}{c}\text { IEEE Bus } \\
\text { System }\end{array}$ & $N_{\text {PMU }}$ & \multicolumn{1}{c}{ Locations of PMUs } & SORI \\
\hline 14-Bus & 3 & $2,6,9$ & 16 \\
\hline 24-Bus & 6 & $2,8,10,15,20,21$ & 29 \\
\hline 30-Bus & 7 & $2,4,10,12,15,19,27$ & 41 \\
\hline 39-Bus & 8 & $3,8,13,16,20,23,25,29$ & 43 \\
\hline 57-Bus & 11 & $1,6,13,19,25,29,32,38,51,54,56$ & 60 \\
\hline & \multirow{3}{*}{$3,8,11,12,17,21,27,31,32,34,37}$, \\
118-Bus & 28 & $40,45,49,52,56,62,72,75,77,80,85$, & 156 \\
& & $86,90,94,102,105,110$ & \\
& & $1,2,3,11,15,17,20,23,24,26,37,41$, \\
& & $43,44,55,57,61,63,70,71,72,77,97$, \\
& & $104,105,108,109,114,119,120,122$, \\
300-Bus & 69 & $126,139,140,145,152,154,155,166$, \\
& & $211,214,216,223,225,229,231,232$, & 393 \\
& \multicolumn{3}{c}{$234,237,238,240,245,249,9002}$, \\
& $9003,9004,9005,9007,9021,9023$, \\
& 9053 & \\
\hline
\end{tabular}

Tables IV and V compared the results between the proposed method and prior studies for the base case and the case considering ZIB, respectively. As can be seen in Table IV, although the number of PMUs required for all bus systems are similar, the SORI values are different. The result for the proposed method is either equal to or has the highest measurement redundancy across all IEEE bus systems, especially in the larger systems. The same thing can be observed in Table V for the case considering ZIB. However, for the IEEE 300-bus system, the proposed method managed to reduce the number of PMUs needed compared to the result obtained in [44] which used the binary semi-definite programming (BSDP) method. 
Table IV Comparison results with prior studies for base case

\begin{tabular}{|c|c|c|c|c|c|c|c|c|c|}
\hline $\begin{array}{l}\text { IEEE bus } \\
\text { system }\end{array}$ & Parameter & $\begin{array}{c}\text { Proposed } \\
\text { Method }\end{array}$ & ES [11] & $\begin{array}{c}\text { Binary } \\
\text { Search [40] }\end{array}$ & DE [17] & BPSO [23] & BPSO [19] & SQP [27] & BSDP [28] \\
\hline 14-bus & SORI & 19 & 19 & 19 & 19 & - & 19 & 19 & 16 \\
\hline 24-bus & $N_{\mathrm{PMU}}$ & 7 & 7 & 7 & - & 7 & - & 7 & - \\
\hline \multirow{2}{*}{ 30-bus } & $N_{\mathrm{PMU}}$ & 10 & 10 & 10 & 10 & 10 & 10 & 10 & 10 \\
\hline & SORI & 52 & 50 & 50 & 52 & 46 & 52 & 48 & 50 \\
\hline 39-bus & $N_{\mathrm{PMU}}$ & 13 & 13 & 13 & 13 & 13 & - & 13 & - \\
\hline 57-bus & SORI & 72 & 68 & - & 72 & 67 & 71 & 71 & 66 \\
\hline \multirow{2}{*}{ 118-bus } & $N_{\mathrm{PMU}}$ & 32 & 32 & - & - & 32 & 32 & 32 & 32 \\
\hline & SORI & 164 & 155 & - & - & 159 & 145 & 163 & 159 \\
\hline \multirow{2}{*}{ 300-bus } & $N_{\mathrm{PMU}}$ & 87 & - & - & - & - & - & - & 87 \\
\hline & SORI & 432 & - & - & - & - & - & - & 423 \\
\hline
\end{tabular}

Table V Comparison results with prior studies for case considering ZIB

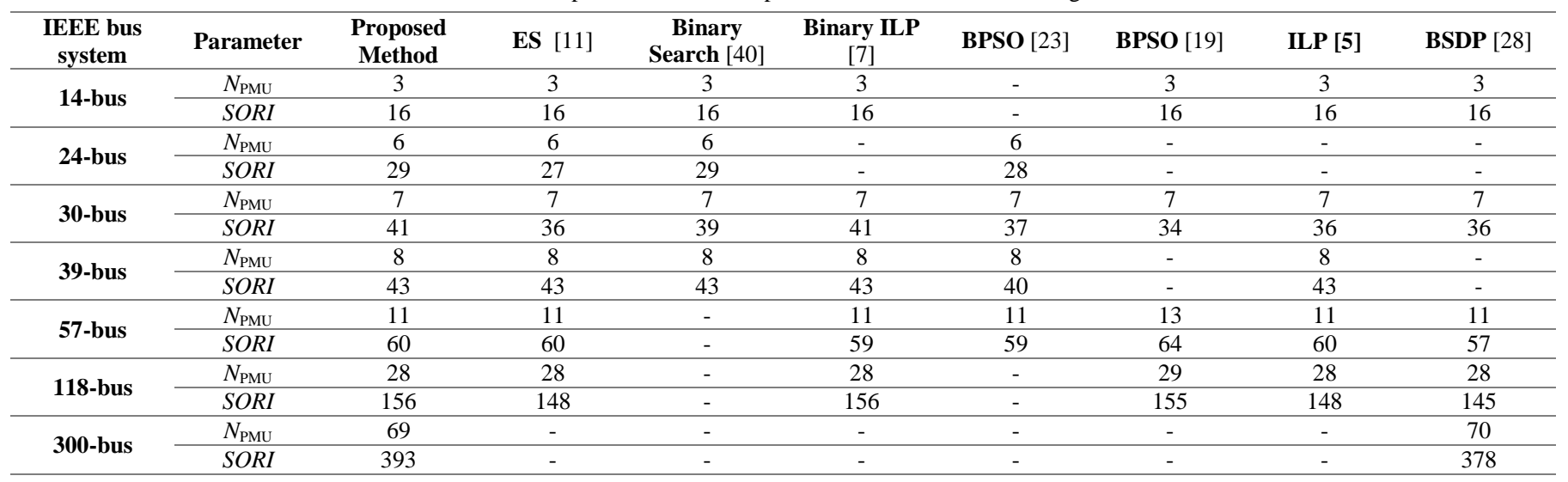

The minimum number of PMUs required for the case considering the single PMU loss is given in Table VI. As can be observed, more PMUs are required to achieve complete observability of the power system compared to the cases with and without considering ZIB. It is expected since at least two PMUs will be responsible for each bus in a power system to ensure the power system remains observable if one of the PMUs becomes malfunction. For instance, for the IEEE 57-bus system, the number of PMUs when single PMU loss is taken

TABLE VI Result for case considering single PMU loss

\begin{tabular}{|c|c|c|c|}
\hline $\begin{array}{l}\text { IEEE } \\
\text { Bus } \\
\text { System }\end{array}$ & $N_{\text {PMU }}$ & Locations of PMUs & SORI \\
\hline 14-Bus & 9 & $2,4,5,6,7,8,9,10,13$ & 39 \\
\hline 24-Bus & 14 & $\begin{array}{l}1,2,3,7,8,9,10,11,15,16,17,20,21, \\
23\end{array}$ & 59 \\
\hline 30-Bus & 21 & $\begin{array}{l}2,3,4,6,7,9,10,11,12,13,15,16,18 \\
20,22,24,25,26,27,28,30\end{array}$ & 85 \\
\hline 39-Bus & 28 & $\begin{array}{l}2,3,6,8,9,10,11,13,14,16,17,19,20, \\
22,23,25,26,29,30,31,32,33,34,35, \\
36,37,38,39\end{array}$ & 96 \\
\hline 57-Bus & 33 & $\begin{array}{l}1,3,4,6,9,11,12,15,19,20,22,24,25, \\
26,28,29,30,32,33,34,36,37,38,41, \\
45,46,47,50,51,53,54,56,57\end{array}$ & 130 \\
\hline 118-Bus & 68 & $\begin{array}{l}2,3,5,6,9,10,11,12,15,17,19,21,22, \\
24,25,27,29,30,31,32,34,35,37,40, \\
42,43,45,46,49,51,52,54,56,57,59, \\
61,62,64,66,68,70,71,73,75,76,77, \\
79,80,83,85,86,87,89,90,92,94,96, \\
100,101,105,106,108,110,111,112, \\
114,116,117\end{array}$ & 309 \\
\hline
\end{tabular}

into account is 33 PMUs. It requires more PMUs than when it is compared to the base case and the case considering ZIB, where the number of PMUs required is 17 PMUs and 11 PMUs, respectively.

For the case considering PMU's channels limit for base case, the number of PMUs obtained is compared with the existing studies and presented in Table VII. As mentioned earlier, by considering PMU's channels limit, the PMU has a finite number of branch lines that it can monitor. Hence, the number of PMUs will be influenced by the limitation. As can be observed, the number of PMUs when using different number of channels is different across all IEEE bus systems. With more channels, the number of PMUs needed is reduced. From Table VII, it can be concluded that the PMUs with 4 number of channels are ample to achieve a complete observability of the power systems with the minimum number of PMUs as obtained in the Table II for the case considering base case.

Next, Table VIII compares the number of PMUs obtained for case considering PMU's channels limit and ZIB with the existing studies. As can be seen from the Table VIII, the number of PMUs needed using the proposed method are comparable with the existing studies that used other optimization methods for all IEEE bus systems. It also must be noted that the number of channels needed is varies for case considering ZIB. For example, for IEEE 24-bus, 30-bus and 39-bus systems, the PMUs with 4 channels are enough to maintain the observability of the power systems with the most minimum number of PMUs, while PMUs with 5 channels are needed for IEEE 14-bus and 
TABLE VII Comparison results for case considering PMU's channels limit for base case

\begin{tabular}{|c|c|c|c|c|c|}
\hline \multirow{2}{*}{$\begin{array}{l}\text { IEEE bus } \\
\text { system }\end{array}$} & \multirow{2}{*}{$\begin{array}{l}\text { Channel } \\
\text { Limit, } L\end{array}$} & \multicolumn{4}{|c|}{ Number of PMUs, $\boldsymbol{N}_{\mathrm{PMU}}$} \\
\hline & & Proposed Method & $\begin{array}{c}\text { Binary Integer } \\
{[41]}\end{array}$ & ILP [42] & ILP [30] \\
\hline \multirow{3}{*}{ 14-bus } & 2 & 7 & 7 & 7 & 7 \\
\hline & 3 & 5 & 5 & 5 & 5 \\
\hline & 5 & 4 & 4 & 4 & 4 \\
\hline \multirow{2}{*}{ 24-bus } & 2 & 12 & - & - & - \\
\hline & 3 & 8 & - & - & - \\
\hline \multirow{4}{*}{ 30-bus } & 2 & 15 & 15 & 15 & 15 \\
\hline & 3 & 11 & 11 & 11 & 11 \\
\hline & 4 & 10 & 10 & 10 & 10 \\
\hline & 5 & 10 & 10 & 10 & 10 \\
\hline \multirow{2}{*}{ 39-bus } & 2 & 21 & - & - & - \\
\hline & 3 & 14 & - & - & - \\
\hline 57-bus & 5 & 17 & 17 & 17 & 17 \\
\hline
\end{tabular}

Table VIII Comparison results for case considering PMU's channels limit and ZIB

\begin{tabular}{|c|c|c|c|c|c|c|c|}
\hline \multirow{2}{*}{$\begin{array}{c}\text { IEEE bus } \\
\text { system }\end{array}$} & \multirow{2}{*}{$\begin{array}{l}\text { Channel } \\
\text { Limit, } L\end{array}$} & \multicolumn{6}{|c|}{ Number of PMUs, $N_{\text {PMU }}$} \\
\hline & & $\begin{array}{l}\text { Proposed } \\
\text { Method }\end{array}$ & ILP [41] & Firefly [15] & ILP [30] & GA [14] & ILP [42] \\
\hline \multirow{4}{*}{ 14-bus } & 2 & 7 & 7 & 7 & 7 & 7 & 7 \\
\hline & 3 & 5 & 5 & 5 & 5 & 5 & 5 \\
\hline & 4 & 4 & 4 & 4 & 4 & 4 & 4 \\
\hline & 5 & 3 & 3 & 3 & 3 & 3 & 3 \\
\hline \multirow{4}{*}{ 24-bus } & 2 & 10 & - & - & - & - & - \\
\hline & 3 & 7 & - & - & - & - & - \\
\hline & 4 & 6 & - & - & - & - & - \\
\hline & 5 & 6 & - & - & - & - & - \\
\hline \multirow{4}{*}{ 30-bus } & 2 & 13 & 12 & 12 & 13 & 12 & 13 \\
\hline & 3 & 8 & 8 & 8 & 9 & 18 & 8 \\
\hline & 4 & 7 & 7 & 8 & 7 & 7 & 7 \\
\hline & 5 & 7 & 7 & 7 & 7 & 7 & 7 \\
\hline \multirow{4}{*}{ 39-bus } & 2 & 14 & 14 & - & - & - & - \\
\hline & 3 & 9 & 9 & - & - & - & - \\
\hline & 4 & 8 & 8 & - & - & - & - \\
\hline & 5 & 8 & 8 & - & - & - & - \\
\hline \multirow{4}{*}{ 57-bus } & 2 & 21 & 21 & 21 & 21 & 21 & 21 \\
\hline & 3 & 14 & 14 & 14 & 14 & 14 & 14 \\
\hline & 4 & 12 & 12 & 13 & 12 & 12 & 12 \\
\hline & 5 & 11 & 11 & 12 & 11 & 11 & 11 \\
\hline
\end{tabular}

IEEE 57-bus systems. However, these results consistent with the results obtained by the existing studies. Therefore, the results proved that the proposed method is capable in solving the OPP problem considering PMU's channels limit using BPSO optimization method, which constraint was never considered before.

\section{CONCLUSION}

This paper proposed a new technique for the BPSO algorithm which integrates a new mutation strategy and V-shaped sigmoid for solving the OPP problem considering ZIB, single PMU loss and PMU's channel limit, which was never considered before in the BPSO algorithm. In addition, this paper also proposed a new fitness function for the case of single PMU loss. The results show that the proposed method can produce a high-quality solution in terms of measurement redundancy compared to prior studies. The proposed method also managed to reduce the number of PMUs needed for IEEE 300-bus system. By applying this technique, particle diversity can be increased and particles can be avoided from being stuck in the local optima.

\section{REFERENCE}

[1] U.S.-Canada Power System Outage Task Force, "Final Report on the August 14, 2003 Blackout in the United States and Canada: Causes and Recommendations.," US FERC Report, 2004. [Online]. Available: http://www.ferc.gov/industries/electric/indus-act/reliability/blackout/ch13.pdf.

[2] A. G. Phadke, J. S. Thorp, and K. J. Karimi, "State Estimation with Phasor Measurements," IEEE Power Eng. Rev., vol. PER-6, no. 2, pp. 48-48, Feb. 1986.

[3] T. L. Baldwin, L. Mili, M. B. Boisen, and R. Adapa, "Power System Observability with Minimal Phasor Measurement Placement," IEEE Trans. Power Syst., vol. 8, no. 2, pp. 707-715, 1993.

[4] N. M. Manousakis, G. N. Korres, and P. S. Georgilakis, "Taxonomy of PMU placement methodologies," IEEE Trans. Power Syst., vol. 27, no. 2, 
pp. 1070-1077, 2012.

[5] F. Aminifar, A. Khodaei, M. Fotuhi-Firuzabad, and M. Shahidehpour "Contingency-Constrained PMU Placement in Power Networks," IEEE Trans. Power Syst., vol. 25, no. 1, pp. 516-523, Feb. 2010.

[6] D. Dua, S. Dambhare, R. K. Gajbhiye, and S. A. Soman, "Optimal multistage scheduling of PMU placement: An ILP approach," IEEE Trans. Power Deliv., vol. 23, no. 4, pp. 1812-1820, 2008.

[7] A. Enshaee, R. A. Hooshmand, and F. H. Fesharaki, "A new method for optimal placement of phasor measurement units to maintain full network observability under various contingencies," Electr. Power Syst. Res., vol. 89, pp. 1-10, Aug. 2012.

[8] N. H. A. Rahman and A. F. Zobaa, "Optimal PMU placement using topology transformation method in power systems," J. Adv. Res., vol. 7, no. 5, pp. 625-634, Sep. 2016.

[9] S. Chakrabarti and E. Kyriakides, "Optimal placement of phasor measurement units for power system observability," IEEE Trans. Power Syst., vol. 23, no. 3, pp. 1433-1440, 2008.

[10] S. Azizi, A. S. Dobakhshari, S. A. Nezam Sarmadi, and A. M. Ranjbar, "Optimal PMU placement by an equivalent linear formulation for exhaustive search," IEEE Trans. Smart Grid, vol. 3, no. 1, pp. 174-182, 2012.

[11]B. K. Saha Roy, A. K. Sinha, and A. K. Pradhan, "An optimal PMU placement technique for power system observability," Int. J. Electr. Power Energy Syst., vol. 42, no. 1, pp. 71-77, 2012.

[12] R. F. Nuqui and A. G. Phadke, "Phasor Measurement Unit Placement Techniques for Complete and Incomplete Observability," IEEE Trans. Power Deliv., vol. 20, no. 4, pp. 2381-2388, Oct. 2005.

[13]Z. Miljanić, I. Djurović, and I. Vujošević, "Optimal placement of PMUs with limited number of channels," Electr. Power Syst. Res., vol. 90, pp. 93 98, 2012.

[14]Z. Miljanić, I. Djurović, and I. Vujošević, "Multiple channel PMU placement considering communication constraints," Energy Syst., vol. 4, no. 2, pp. 125-135, 2013.

[15]K. Arul jeyaraj, V. Rajasekaran, S. K. Nandha kumar, and K. Chandrasekaran, "A Multi-objective Placement of Phasor Measurement Units Considering Observability and Measurement Redundancy using Firefly Algorithm," J. Electr. Eng. Technol., vol. 10, no. 2, pp. 474-486, Mar. 2015.

[16]J. Peng, Y. Sun, and H. F. Wang, "Optimal PMU placement for full network observability using Tabu search algorithm," Int. J. Electr. Power Energy Syst., vol. 28, no. 4, pp. 223-231, 2006.

[17] A. H. Al-Mohammed, M. A. Abido, and M. M. Mansour, "Optimal placement of synchronized phasor measurement units based on differential evolution algorithm," in 2011 IEEE PES Conference on Innovative Smart Grid Technologies - Middle East, 2011, pp. 1-9.

[18]C. Peng, H. Sun, and J. Guo, "Multi-objective optimal PMU placement using a non-dominated sorting differential evolution algorithm," Int. J. Electr. Power Energy Syst., vol. 32, no. 8, pp. 886-892, Oct. 2010.

[19]A. Ahmadi, Y. Alinejad-Beromi, and M. Moradi, "Optimal PMU placement for power system observability using binary particle swarm optimization and considering measurement redundancy," Expert Syst. Appl., vol. 38, no. 6, pp. 7263-7269, 2011.

[20] S. Chakrabarti, G. K. Venayagamoorthy, and E. Kyriakides, "PMU placement for power system observability using binary particle swarm optimization,' in Power Engineering Conference, 2008. AUPEC '08. Australasian Universities, 2008, pp. 1-5.

[21]M. Hajian, A. M. Ranjbar, T. Amraee, and A. R. Shirani, "Optimal placement of phasor measurement units: Particle swarm optimization approach," in 2007 International Conference on Intelligent Systems Applications to Power Systems, 2007, no. 1, pp. 1-6.

[22]M. Hajian, A. M. Ranjbar, T. Amraee, and B. Mozafari, "Optimal placement of PMUs to maintain network observability using a modified BPSO algorithm," Int. J. Electr. Power Energy Syst., vol. 33, no. 1, pp. 28 34, 2011.

[23]Z. H. Rather, C. Liu, Z. Chen, and P. Thogersen, "Optimal PMU Placement by improved particle swarm optimization," in 2013 IEEE Innovative Smart Grid Technologies-Asia (ISGT Asia), 2013, pp. 1-6.

[24]C. Sharma and B. Tyagi, "An approach for optimal PMU placement using binary particle swarm optimization with conventional measurements," Int. J. Eng. Sci. Technol., vol. 3, no. 3, 2011.

[25] J. m. Wang, C. Li, and J. Zhang, "Optimal Phasor Measurement Unit Placement by an Improved PSO Algorithm," 2012 Asia-Pacific Power and Energy Engineering Conference. pp. 1-4, 2012.

[26]Y. del Valle, G. K. Venayagamoorthy, S. Mohagheghi, J.-C. Hernandez, and R. G. Harley, "Particle Swarm Optimization: Basic Concepts, Variants and Applications in Power Systems," IEEE Trans. Evol. Comput., vol. 12, no. 2, pp. 171-195, 2008.

[27]N. P. Theodorakatos, N. M. Manousakis, and G. N. Korres, "A sequential quadratic programming method for contingency-constrained phasor measurement unit placement," Int. Trans. Electr. Energy Syst., 2014.

[28] J. Löfberg, N. M. Manousakis, G. N. Korres, and T. C. Xygkis, "Optimal phasor measurement unit placement for numerical observability in the presence of conventional measurements using semi-definite programming," IET Gener. Transm. Distrib., vol. 9, no. 15, pp. 2427-2436, 2015.

[29]A. Mahari and H. Seyedi, "Optimal PMU placement for power system observability using BICA, considering measurement redundancy," Electr. Power Syst. Res., vol. 103, pp. 78-85, 2013.

[30] M. Korkali and A. Abur, "Placement of PMUs with channel limits," 2009 IEEE Power and Energy Society General Meeting, PES '09. pp. 1-4, 2009.

[31] Y. Duan, R. G. Harley, and T. G. Habetler, "Comparison of Particle Swarm Optimization and Genetic Algorithm in the design of permanent magnet motors," in 2009 IEEE 6th International Power Electronics and Motion Control Conference, 2009, vol. 3, pp. 822-825.

[32] M. Clerc and J. Kennedy, "The particle swarm - explosion, stability, and convergence in a multidimensional complex space," IEEE Trans. Evol. Comput., vol. 6, no. 1, pp. 58-73, Feb. 2002.

[33] J. Kennedy and R. C. Eberhart, "A discrete binary version of the particle swarm algorithm," in 1997 IEEE International Conference on Systems, Man, and Cybernetics. Computational Cybernetics and Simulation, 1997, vol. 5, pp. 4-8.

[34] S. Mirjalili and A. Lewis, "S-shaped versus V-shaped transfer functions for binary Particle Swarm Optimization," Swarm Evol. Comput., vol. 9, pp. 114, Apr. 2013.

[35]G.-C. Luh, C.-Y. Lin, and Y.-S. Lin, "A binary particle swarm optimization for continuum structural topology optimization," Appl. Soft Comput., vol. 11, no. 2, pp. 2833-2844, Mar. 2011.

[36]H. Nezamabadi-pour, M. Rostami-Shahrbabaki, and M. MaghfooriFarsangi, "Binary particle swarm optimization: challenges and new solutions," CSI J Comput Sci Eng, vol. 6, no. 1-A, pp. 21-32, 2008.

[37]Q. Ni and J. Deng, "Analysis of population diversity of dynamic probabilistic particle swarm optimization algorithms," Math. Probl. Eng., vol. 2014, 2014.

[38] T. K. Maji and P. Acharjee, "Multiple solutions of optimal PMU placement using exponential binary PSO algorithm," in 2015 Annual IEEE India Conference (INDICON), 2015, pp. 1-6.

[39] C. Yuehui, C. Haiyan, C. Jinfu, and D. Xianzhong, "An Improved Particle Swarm Optimization Algorithm for Multistage and Coordinated Planning of Transmission Systems," in Transmission and Distribution Conference and Exhibition: Asia and Pacific, 2005 IEEE/PES, 2005, pp. 1-6.

[40]S. Chakrabarti and E. Kyriakides, "Optimal Placement of Phasor Measurement Units for Power System Observability," IEEE Trans. Power Syst., vol. 23, no. 3, pp. 1433-1440, Aug. 2008.

[41]R. Kumar and V. S. Rao, "Optimal placement of PMUs with limited number of channels," in 2011 North American Power Symposium, 2011, no. 1 , pp. 1-7.

[42]N. Fan and J. P. Watson, "On integer programming models for the multichannel PMU placement problem and their solution," Energy Syst., vol. 6, no. 1, 2014. 